\title{
Design Optimization of a Scramjet Vehicle for Ascent Using Surrogate Optimization
}

\author{
Derek J. Dalle* and James F. Driscoll ${ }^{\dagger}$ \\ University of Michigan, Ann Arbor, MI 48109
}

\begin{abstract}
Design optimization is performed for a scramjet-powered vehicle that ascends along a trajectory with constant dynamic pressure from Mach 7 to Mach 13. The net acceleration is free during the trajectory, and the optimum acceleration profile is dependent on the vehicle design. To perform this analysis, a new reduced-order model (MASIV) is used that can evaluate the forces and moments on the vehicle in about five seconds on a single computer while accounting for wave interactions, fuel-air mixing, finite-rate chemistry, and other complex physical phenomena. It is found that the optimum acceleration is close to the maximum acceleration that the vehicle can attain. To minimize the fuel consumed for the entire ascent trajectory, there is a trade-off between performance and robustness to variations in angle of attack.
\end{abstract}

\section{Introduction}

Among the applications of an air-breathing hypersonic vehicle, a common phase of flight is acceleration from one flight Mach number to a higher flight Mach number. While it is possible to design a cruiser to operate at a single velocity throughout the flight if it is accelerated to its cruise condition by other means of propulsion, hypersonic cruise vehicles typically perform part of the acceleration using the ramjet or scramjet propulsion system to reduce total fuel consumption. Furthermore, the optimum cruise Mach number may increase throughout the flight as the vehicle burns fuel and becomes lighter. For a hypersonic vehicle designed for access to space, acceleration is the primary objective of the mission.

Previously, the optimization of the ascent of a hypersonic vehicle was performed by Calise et al. [1], Corban et al. [2], Powell et al. [3], Olds and Budianto [4], McGinnis [5], and Lu [6,7]. Their vehicle models use empirical or algebraic formulas for combustion and inlet efficiencies, and ignored important couplings such as the sensitivity of thrust to angle of attack. They employed some unrealistic assumptions, for example that lift is proportional to angle of attack. Except for [3], these previous studies did not account for trim drag. For many vehicles, such as the geometry used in this work, an increase in thrust raises the pressure on the nozzle surface, which contributes directly to lift. This is just one example of a coupling that was ignored in many previous hypersonic vehicle ascent studies.

Because of the integration among the various parts of hypersonic vehicles, optimization considering several disciplines of vehicle performance and modeling (for example propulsion and flight dynamics) is highly relevant. This technique is called multidisciplinary design optimization (MDO), and it has been applied to hypersonic vehicles [8].

Many objective functions have been used for design optimization of hypersonic vehicles. The optimization is usually tailored to the mission for which the vehicle is intended. For transport vehicles, air-breathing range is an ideal objective function [8]. Fuel consumption at a cruise condition can be used as an approximation. However, compared to low-speed aircraft, hypersonic vehicles have more fuel as a fraction of vehicle mass. As a result, the lift-to-drag ratio varies significantly throughout the mission, and varying $C_{L} / C_{D}$ has significant impact on the range, for example [9].

Some authors [10-12] have also considered the challenging problem of multiobjective optimization. Optimization with multiple objectives is often difficult to interpret, but it can be used to identify tradeoffs in

\footnotetext{
${ }^{*}$ Graduate Research Assistant, Department of Aerospace Engineering, AIAA Member, dalle@umich . edu

${ }^{\dagger}$ Professor, Department of Aerospace Engineering, AIAA Fellow, james fd@umich . edu
} 


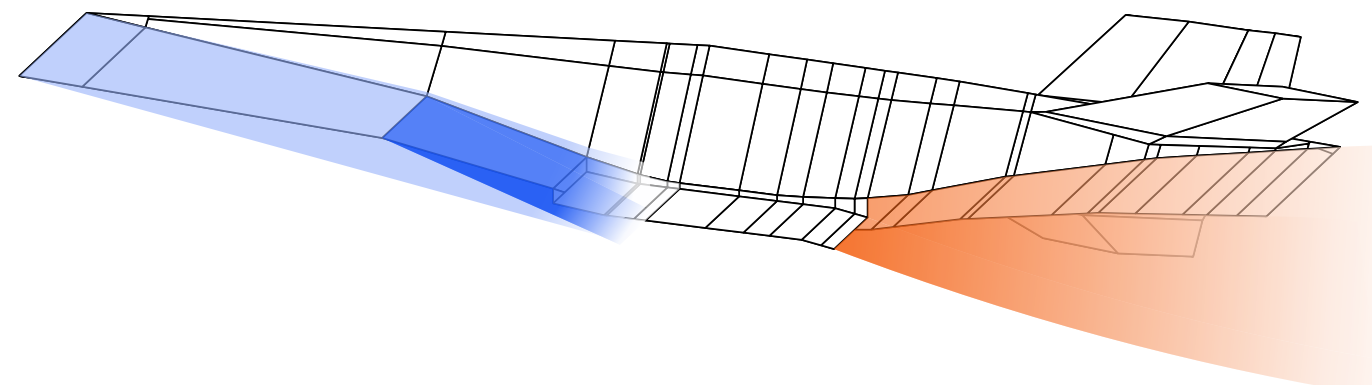

Figure 1. View of MAX-1 vehicle with stylized shocks and exhaust plume.

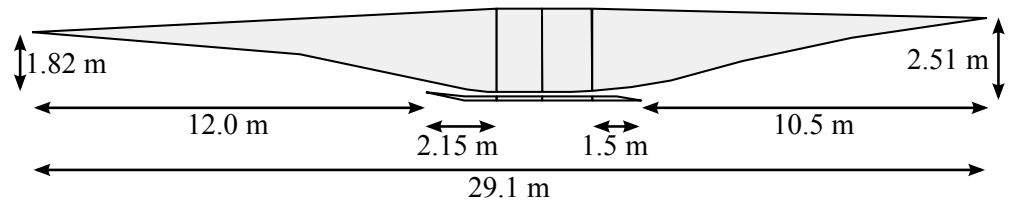

a) Inlet and nozzle dimensions.

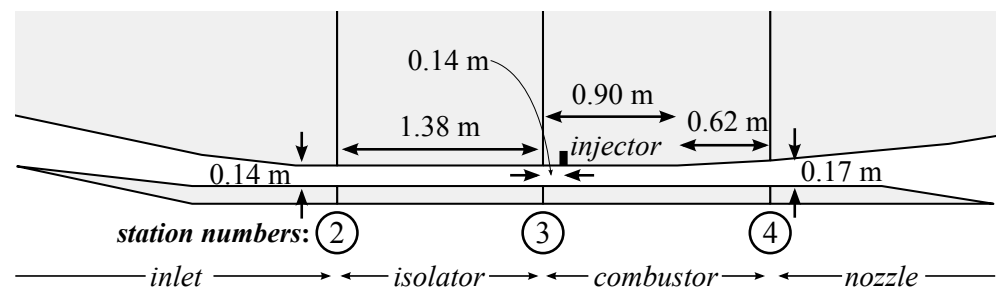

b) Internal flowpath dimensions with station numbers.

Figure 2. MAX-1 engine flowpath dimensions. Engine width is 2.143 m.

the design space.

Much of the literature on optimization in the field of hypersonics focuses on the design of individual components, often at individual operating conditions. Smart [13] demonstrated a highly useful algorithm for optimizing two-dimensional scramjet inlets under just those assumptions. With the ability to rapidly compute performance at off-design conditions, we showed that such inlet designs have very poor performance for even small perturbations from the design conditions [14, 15]. Brown et al. [16] discussed robust optimization to mitigate this result. In $[15,17]$, we present an approach to the inlet design problem that addresses these issues directly with constraints on shock interactions. However, an inlet with fewer shock interactions and less drag may not necessarily result in better overall performance of the vehicle.

The primary goal of this work is to identify changes to the vehicle design that reduce the total fuel consumption required to accelerate from Mach 7 to Mach 13. The assumption of constant dynamic pressure is maintained for two reasons: the engine tends to prefer lower altitudes (higher dynamic pressures) because the higher air density leads to increased thrust, but there is a limit to how much dynamic pressure the structure can withstand [2]. At high Mach numbers the constraint on altitude will be aerodynamic heating, which is proportional to $\rho V^{3}$, but that constraint is not considered in this work.

To perform the design optimization in this work, a parametrized geometry is used. The baseline geometry is shown in Figs. 1 and 2, but changes to the geometry can be made using 40 design variables. For each vehicle configuration, an operating map is made that tabulates the required control variables to trim the vehicle for each combination of Mach number, acceleration, and fuel mass. Then the optimum acceleration 


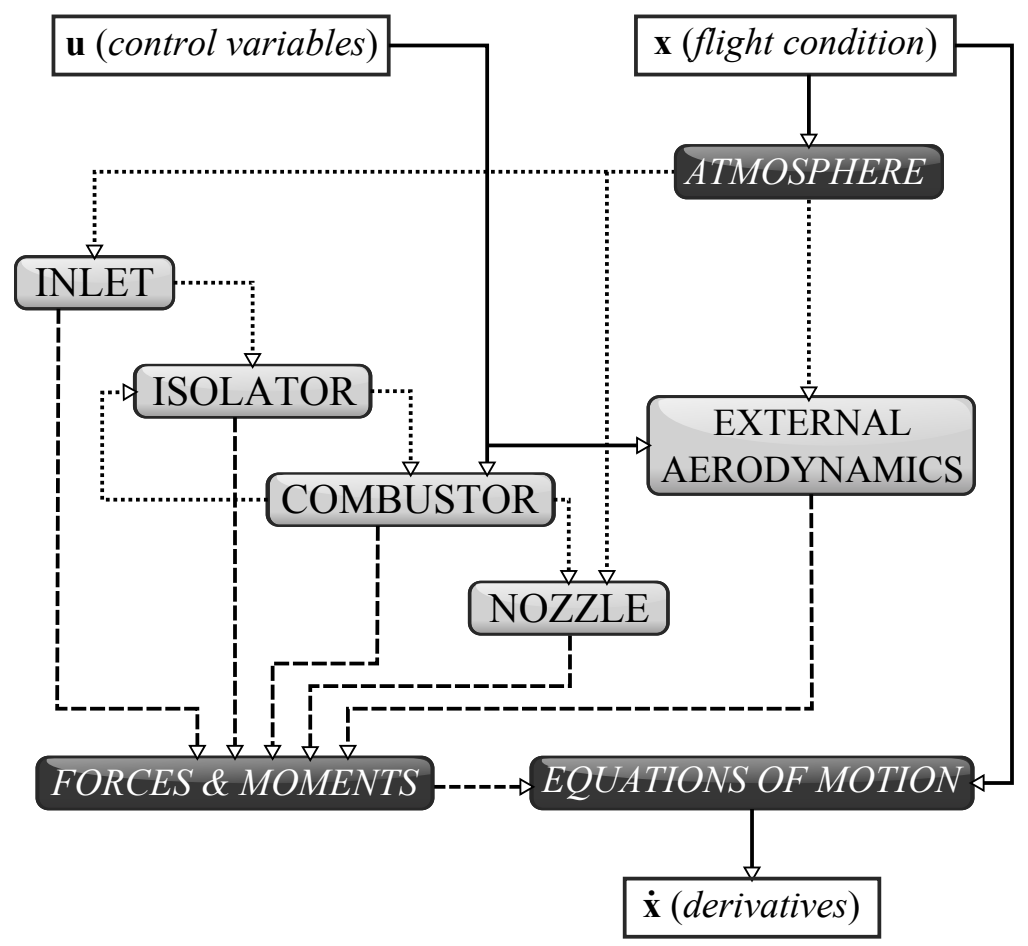

Figure 3. Overview of information flow within the MASTrim program. White blocks represent inputs and outputs, and solid lines show the flow of information to and from these inputs and outputs. Dark blocks represent models not specific to the vehicle, and the gray blocks represent vehicle component models. Dotted lines show information about gas states, and dashed lines show force $\&$ moment information.

as a function of Mach number is calculated using the operating map as a surrogate.

The layout of the remainder of the paper is as follows. Section II provides an overview of the Michigan/AFRL Scramjet In Vehicle (MASIV) model used for this work. Section III describes a procedure for optimizing the acceleration profile along an ascent trajectory with constant dynamic pressure, while the effects of various changes to the design on both performance and stability are investigated in Sec. IV. Finally, results of the design optimization are presented in Secs. V and VI.

\section{Dual-Mode Ramjet-Scramjet Vehicle Model}

The MASIV/MASTrim model has been the subject of many previous papers [14,17-22] and is used for this work. Figure 1 illustrates the generic hypersonic vehicle design used for the present work, and the name "MAX-1" is associated with this vehicle. The propulsive system of the MAX-1 is approximately the same size as the vehicle itself, and can be broken into four components.

An inlet provides both lift for the vehicle to maintain level flight and compression to ensure efficient combustion. This is followed by a constant-area isolator section that separates the inlet from the combustor area where the fuel injection and heat addition occurs. Finally, the exhaust products are allowed to expand in an external nozzle area. These components and the respective dimensions for the MAX-1 vehicle are shown in Fig. 2. The mass properties of the MAX-1 vehicle are given in Table 1, and sample solutions from the inlet and nozzle are shown in Fig. 4.

Figure 3 shows how information is passed to and from each component in the MASTrim code. In Fig. 3, there are three types of blocks and three types of arrows, and the information conveyed by these symbols is explained in the caption.

The inputs to the simulation consist of states ( $\mathbf{x}$, also referred to as the flight condition) and a vector of control variables $(\mathbf{u})$. The flight condition is fed into an atmospheric model that determines the freestream 


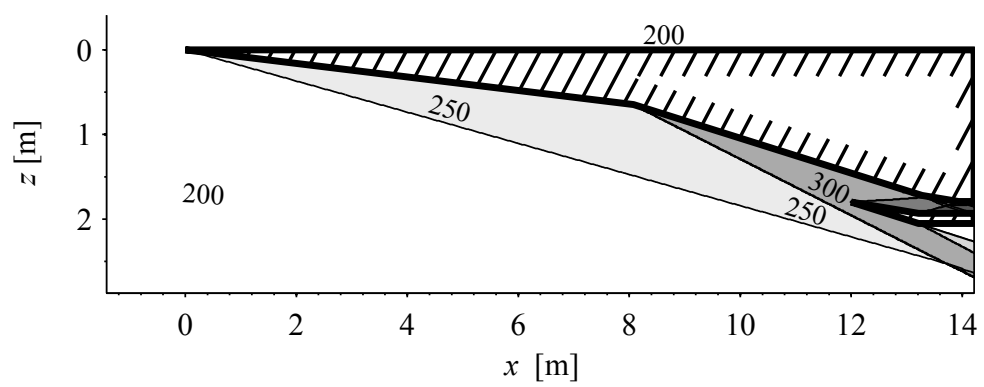

a) Inlet

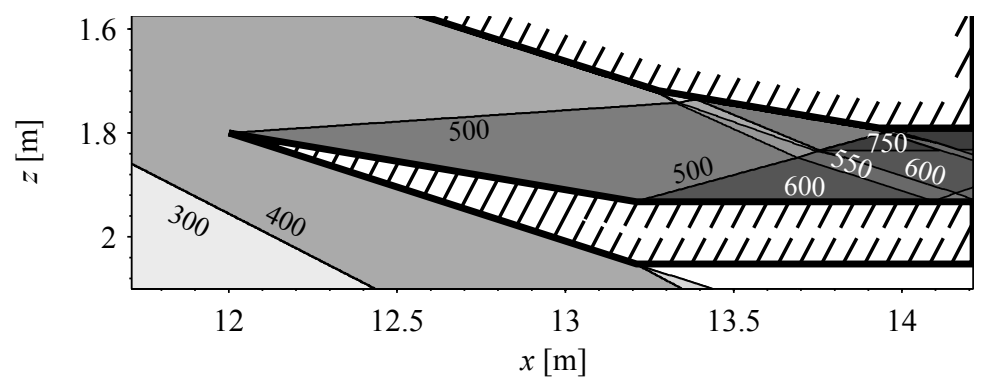

b) Inlet cowl region

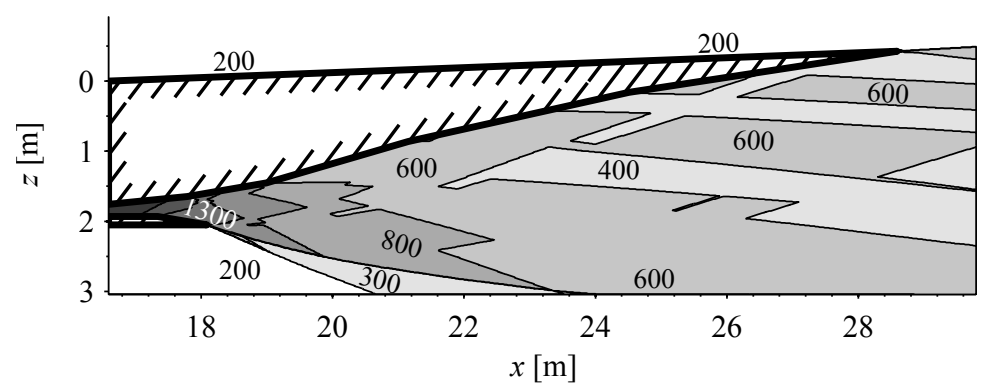

c) Nozzle

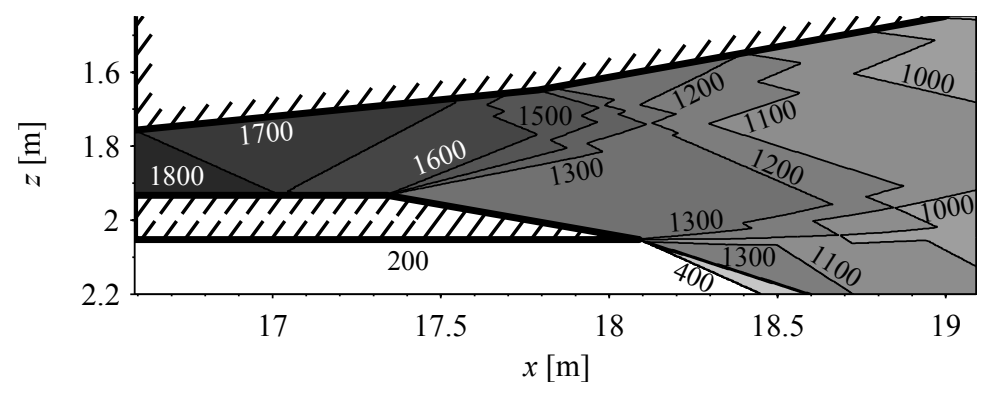

d) Nozzle cowl region

Figure 4. Contours of temperature in Kelvins at a trimmed Mach 8 flight condition calculated using SAMURI. 
Table 1. Mass properties of vehicle.

\begin{tabular}{ccc}
\hline \hline Symbol & Name & Value \\
\hline & operational empty mass & $1.93 \times 10^{4} \mathrm{~kg}$ \\
& maximum fuel mass & $2.38 \times 10^{4} \mathrm{~kg}$ \\
$I_{x x}$ & specific moment of inertia & $2.33 \mathrm{~m}^{2}$ \\
$I_{y y}$ & specific moment of inertia & $36.23 \mathrm{~m}^{2}$ \\
$I_{z z}$ & specific moment of inertia & $37.90 \mathrm{~m}^{2}$ \\
$I_{x z}$ & specific product of inertia & $0.54 \mathrm{~m}^{2}$ \\
$x_{c g}$ & center of mass location & $-12.88 \mathrm{~m}$ \\
$z_{c g}$ & center of mass location & $0.17 \mathrm{~m}$ \\
\hline \hline
\end{tabular}

air conditions needed for the inlet and external aerodynamics solvers. The four engine components are shown at left in a diagonal pattern. The force and moment created by each of the four engine components is calculated individually, as indicated by the dashed lines in Fig. 3. External pressures and viscous effects naturally provide another source of forces on the vehicle, and this is represented by the fifth input to the forces \& moments block.

Finally, the outputs of the model consist mostly of accelerations and angular accelerations. Calculating these requires more than simply dividing the net forces by the mass or multiplying the inverse of the inertia tensor by the net moments. Instead, the six-degree-of-freedom equations of motion for a rotating ellipsoidal Earth are used.

\section{Accelerating Trajectory Optimization}

We define an altitude profile in the velocity in the velocity-altitude plane as a function

$$
h=h(V)
$$

such that the altitude is determined as a function of $V$, the magnitude of the velocity of the vehicle (alternatively, $h=h(M))$. In this work, the function $h(V)$ is selected so that the dynamic pressure,

$$
q=\frac{1}{2} \rho V^{2}=\frac{\gamma}{2} p M^{2}
$$

is held constant, but other functions $h(V)$ could be selected. This function $h(V)$ does not uniquely determine a trajectory because other variables, such as acceleration $(a)$ can still vary. In other words, there is a function

$$
a=a(V)
$$

that prescribes the acceleration for each velocity.

An example of such a trajectory is shown in Fig. III. The goal of this trajectory optimization is to determine the function $a(V)$ that minimized fuel consumption for the entire ascent.

\section{A. Trajectory Closure}

The altitude profile leads to a relationship between acceleration and flight path angle $(\gamma)$. Differentiating $h(V)$ with respect to time using the chain rule gives

$$
\dot{h}=\frac{\mathrm{d} h}{\mathrm{~d} V} \frac{\mathrm{d} V}{\mathrm{~d} t}=h^{\prime}(V) a
$$

Since $\dot{h}=V \sin \gamma$, we can solve for the flight path angle.

$$
\sin \gamma=\frac{\mathrm{d} h}{\mathrm{~d} V} \frac{a}{V}
$$



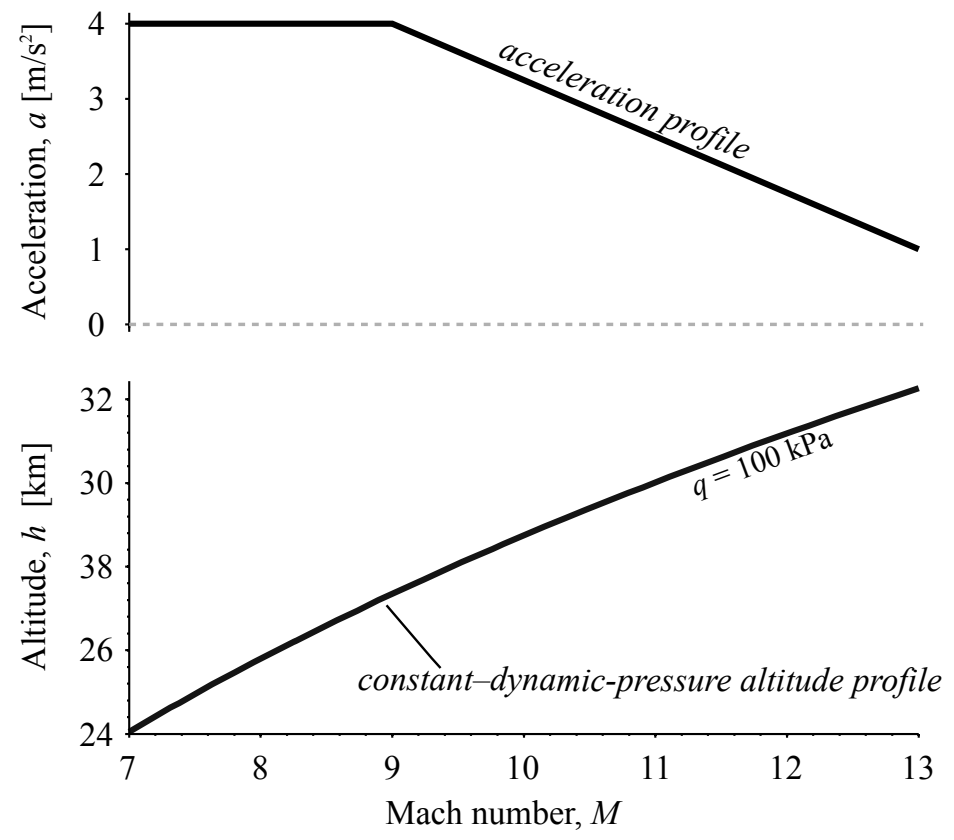

Figure 5. Example trajectory defined by altitude profile, $h(V)$, and acceleration profile, $a(V)$.

This means that the flight path angle (or climb rate) is determined by our two functions $h(V)$ and $a(V)$. There is also a question of $\dot{\gamma}$, which we can find by taking another derivative of the altitude.

$$
\begin{aligned}
\ddot{h} & =\frac{\mathrm{d}}{\mathrm{d} t}(V \sin \gamma)=a \sin \gamma+V \dot{\gamma} \cos \gamma \\
& =\frac{\mathrm{d}}{\mathrm{d} t}\left(\frac{\mathrm{d} h}{\mathrm{~d} V} \frac{\mathrm{d} V}{\mathrm{~d} t}\right)=\frac{\mathrm{d}^{2} h}{\mathrm{~d} V^{2}} a^{2}+\frac{\mathrm{d} h}{\mathrm{~d} V} \frac{\mathrm{d} a}{\mathrm{~d} t}
\end{aligned}
$$

Equating the two expressions for $\ddot{h}$ and solving for $\dot{\gamma} \cos \gamma$ gives

$$
\dot{\gamma} \cos \gamma=\frac{1}{V}\left[\frac{\mathrm{d}^{2} h}{\mathrm{~d} V^{2}} a^{2}+\frac{\mathrm{d} h}{\mathrm{~d} V} \frac{\mathrm{d} a}{\mathrm{~d} t}-a \sin \gamma\right]
$$

Noting that

$$
\frac{\mathrm{d} a}{\mathrm{~d} t}=\frac{\mathrm{d} a}{\mathrm{~d} V} \frac{\mathrm{d} V}{\mathrm{~d} t} \quad \sin \gamma=\frac{a}{V} \frac{\mathrm{d} h}{\mathrm{~d} V}
$$

this equation can be simplified to

$$
\dot{\gamma} \cos \gamma=\left[\frac{\mathrm{d}^{2} h}{\mathrm{~d} V^{2}} a+\left(\frac{\mathrm{d} a}{\mathrm{~d} V}-\frac{a}{V}\right) \frac{\mathrm{d} h}{\mathrm{~d} V}\right] \frac{a}{V}
$$

Each of the terms in Eq. (6) is very small, and we usually just set $\dot{\gamma}=0$ during trajectory simulation. Exceptions include very steep climbs $(\cos \gamma \approx 0$ and $\mathrm{d} h / \mathrm{d} V \gg 0)$ and sharp changes in the altitude profile $\left(\mathrm{d}^{2} h / \mathrm{d} V^{2} \gg 0\right)$.

The dynamic pressure is $q=\frac{1}{2} \rho V^{2}$ where $\rho$ is given as a function of altitude $(\rho(h))$ given by an atmosphere model, e.g. [23]. Differentiating the dynamic pressure with respect to altitude allows one to solve for the derivative of altitude with respect fo velocity:

$$
\frac{\mathrm{d} h}{\mathrm{~d} V}=-\frac{2 \rho}{V \frac{\mathrm{d} \rho}{\mathrm{d} h}}=-\frac{2 \rho}{V \rho^{\prime}(h)}
$$

$$
6 \text { of } 16
$$


Substituting this into Eq. (5) gives

$$
\sin \gamma=-\frac{2 \rho a}{V^{2} \rho^{\prime}(h)}
$$

\section{B. Fuel Consumption}

One appropriate objective for a trajectory that must connect two fixed flight conditions is the total fuel usage. In this section we describe a method to calculate the total fuel consumption for a trajectory without directly considering time. Similar approaches can be found in [7], in which the equations of motion were written in terms of downrange distance, and [1], where it was shown that the fuel consumption could be calculated using energy as a variable of integration. In the present work, we use velocity as the dependent variable and eliminate time completely from the simulation. If time is used as a variable, the total fuel used is defined as

$$
m_{f}(t)=\int_{0}^{t} \dot{m}_{f}\left(V(\tau), a(\tau), m_{f}(\tau)\right) \mathrm{d} \tau
$$

Suppose that a given trajectory is parametrized by $n$ pairs of flight conditions $\left(M_{1}, a_{1}\right), \ldots,\left(M_{n}, a_{n}\right)$, and let $V_{1}, \ldots, V_{n}$ be the velocities corresponding to the Mach numbers $M_{1}, \ldots, M_{n}$. A simple change of variables transforms Eq. (9) into

$$
m_{f}=\int_{V_{1}}^{V_{n}} \frac{\dot{m}_{f}\left(V, a(V), m_{f}(V)\right)}{a(V)} \mathrm{d} V
$$

Using trapezoidal numerical integration, this integral can be estimated as

$$
m_{f}=\sum_{i=1}^{n-1} \frac{1}{2}\left(\frac{\dot{m}_{f, i}}{a_{i}}-\frac{\dot{m}_{f, i+1}}{a_{i+1}}\right)\left(V_{i+1}-V_{i}\right)
$$

This formulation allows the total fuel consumption to be calculated without a time-domain simulation, but it may still be useful to calculate the time spent on each trajectory segment. In this case a second-order approximation is

$$
\Delta t_{i}=t_{i+1}-t_{i}=2 \frac{V_{i+1}-V_{i}}{a_{i+1}+a_{i}}
$$

\section{Example Operating Map}

Consider a trajectory in which a vehicle accelerates from Mach 7 to Mach 13 with a constant dynamic pressure of $100 \mathrm{kPa}$. Figure 6 shows two operating maps for the flight conditions along this trajectory with an acceleration between 0 and $6 \mathrm{~m} / \mathrm{s}^{2}$. The first map (sold black contour lines) is for the case that the vehicle has full fuel tanks. The dashed gray lines show the trim conditions for the case that the fuel tanks are empty. The difference in mass of the vehicle is substantial (more than 50\%, see Table 1), and as a result the fully fueled vehicle has a higher trim angle of attack. The higher angle of attack results in more drag and approximately twice as much fuel consumption.

\section{Trajectory Optimization Results}

Figure 7 shows the equivalence ratio $(E R)$ and mass fraction of oxygen in the exhaust $\left(Y_{\mathrm{O}_{2}, 5}\right)$ for an optimized trajectory for the MAX-1 baseline vehicle flying at a constant dynamic pressure of $100 \mathrm{kPa}$. The results of several trajectories with constant acceleration are shown in light gray lines for comparison.

It can be seen in Fig. 7a that the optimum trajectory is at or near the maximum acceleration that the vehicle can sustain at each Mach number. (The fact that there are no gray lines above the optimum trajectory indicates that the thick black optimum trajectory curve is the maximum acceleration.) However, the maximum acceleration does not occur at an equivalence ratio of approximate 1.0. Operating at an equivalence 


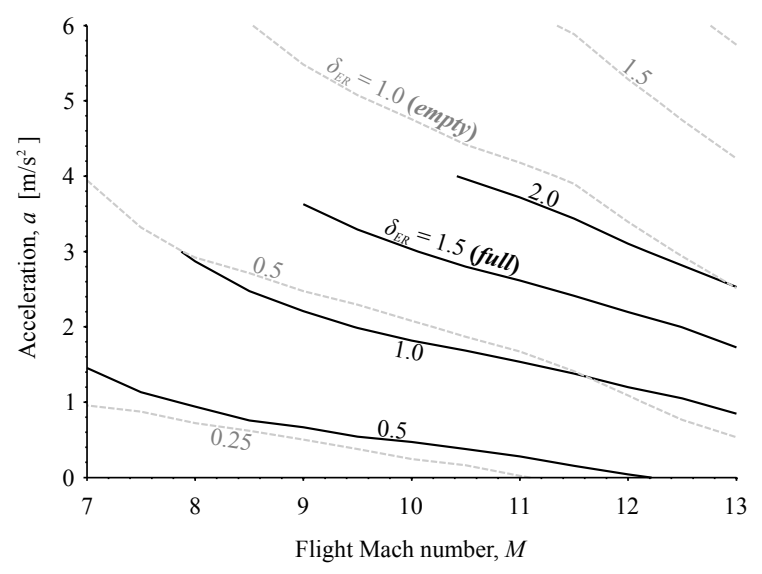

a) Equivalence ratio $\left(\delta_{E R}\right)$.

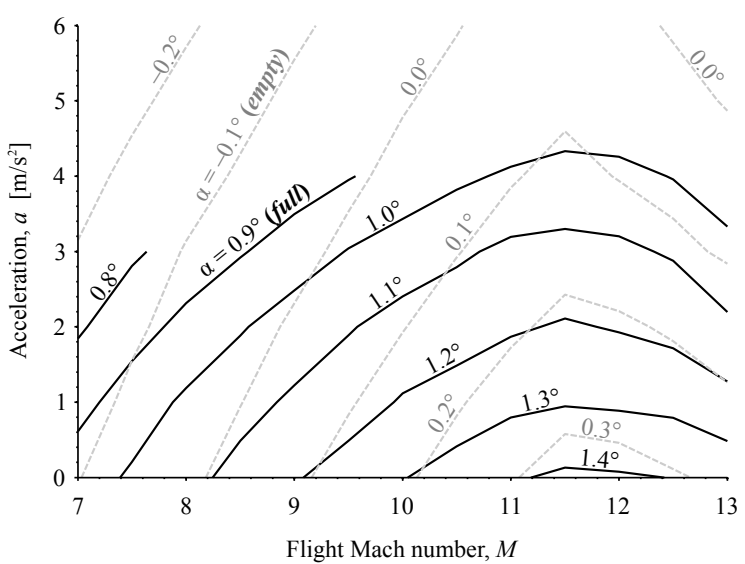

b) Angle of attack $(\alpha)$.

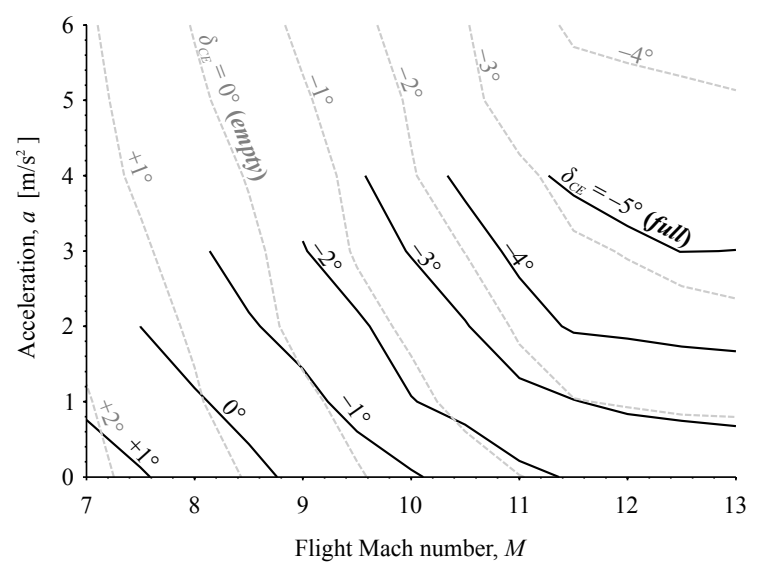

c) Elevon angle $\left(\delta_{C E}\right)$. A trailing-edge-down deflection is positive.

Figure 6. Trimmed flight conditions with $q=100 \mathrm{kPa}$. Solid black lines are for full fuel tanks and dashed gray lines are for empty fuel tanks.

ratio of unity indicates that exactly enough fuel is injected into the combustor flow so that if all the fuel burned it would use up all of the oxygen flowing into the combustor. However, combustion efficiencies are not guaranteed to be near $100 \%$, which is to say that there is no guarantee that all of the fuel injected into the combustor will burn.

At Mach 12, if the vehicle has an acceleration of $2 \mathrm{~m} / \mathrm{s}^{2}$, Fig. 7a shows that the equivalence ratio required to balance the thrust and drag on the vehicle is 1.0. However, Fig. $7 \mathrm{~b}$ shows that the exhaust still contains $6.2 \%$ oxygen by mass. This indicates that only about two thirds of the fuel is burning. Furthermore, since there is still oxygen available for combustion, injecting more fuel continues to increase the acceleration, which results in equivalence ratios greater than unity.

Figure $7 \mathrm{~b}$ shows that above Mach 9, the optimum trajectory injects enough fuel so that nearly all of the oxygen is used for combustion. However, at lower Mach numbers there is still molecular oxygen in the exhaust even at the maximum acceleration. The reason is that the heat addition in the combustor drives the Mach number toward unity. Adding more fuel causes thermal choking in the engine, which causes the engine to transition into ram-mode operation, which is not considered here. However, ram-mode flight is practical for a vehicle of the type considered in this work, so allowing for a mode transition during the trajectory is an important aspect of this flight regime and is discussed in Sec. VI. 


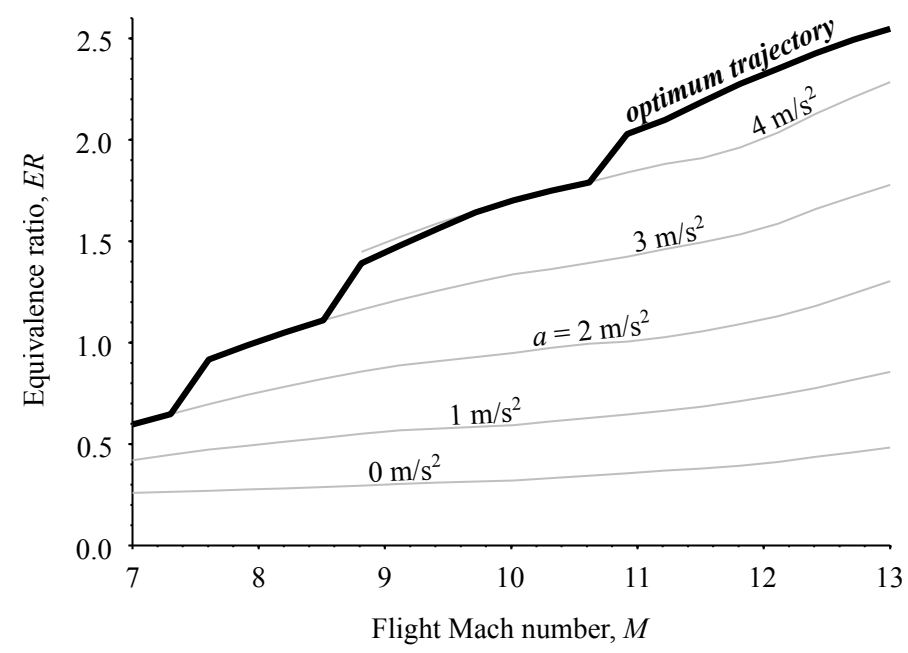

a) Equivalence ratio $(E R)$.

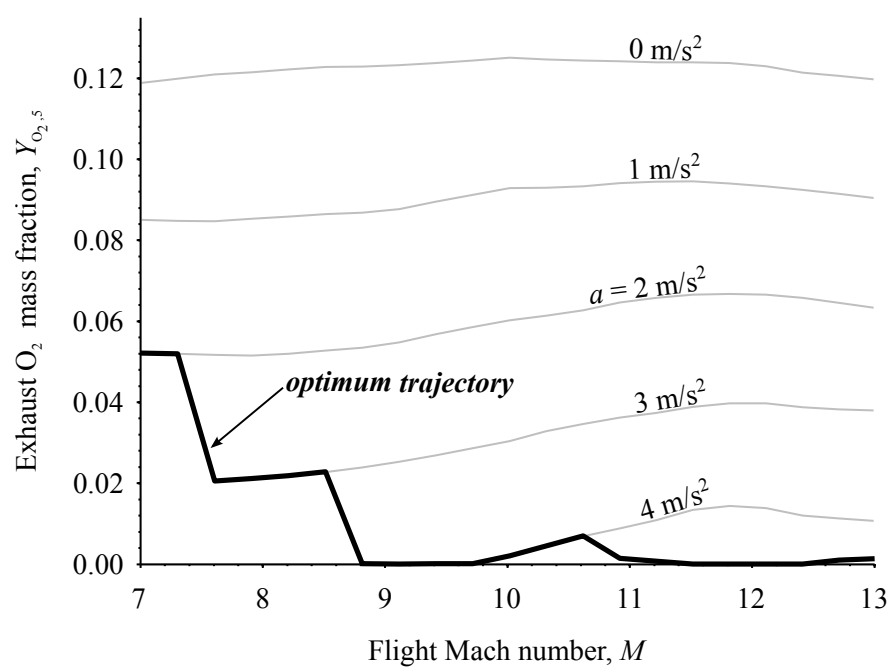

b) Combustor exhaust $\mathrm{O}_{2}$ mass fraction $\left(Y_{\mathrm{O}_{2}, 5}\right)$.

Figure 7. State variables along minimum-fuel trajectory and constant-acceleration trajectories.

\section{Sensitivities to Design Parameters}

Table 2 describes ten vehicle designs for which optimized ascent trajectories were computed. The total fuel consumption for each of the vehicles are listed in Table 3.

Because the initial mass of the vehicle was not constant for all the designs, a figure of merit was introduced that differs slightly from the total mass of fuel consumed. This figure of merit is called the trajectory specific impulse, and its equation is

$$
I_{s p}=\frac{V_{2}-V_{1}}{g \ln \left(m_{1} /\left(m_{1}-m_{f}\right)\right)}
$$

where $m_{1}$ is the initial mass of the vehicle, and $m_{f}$ is the total fuel consumption. This equation gives the specific impulse of a rocket-powered vehicle having the same initial mass $\left(m_{1}\right)$ that achieved the same change in velocity $\left(V_{2}-V_{1}\right)$ using the same amount of fuel $\left(m_{f}\right)$. This figure of merit is a function of the ratio of fuel consumed to total vehicle mass $\left(m_{f} / m_{1}\right)$, and thus it provides a metric that is roughly independent of vehicle size. In Table 3, it can be seen that the vehicle that is identical to the baseline MAX-1 vehicle except 
Table 2. Description of changes to default vehicle considered for sensitivity analysis.

\begin{tabular}{ll}
\hline \hline Vehicle Label & Description \\
\hline MAX-1 & Baseline vehicle, as shown in Fig.s 1 and 2 \\
$r_{f}=0.6$ & Same vehicle geometry but with fuel tanks $10 \%$ more full \\
$\Delta x_{c g}=0.05$ & Center of gravity shifted backward by $5 \%$ of vehicle length \\
$\Delta x_{c g}=0.025$ & Center of gravity shifted backward by $2.5 \%$ of vehicle length \\
$r_{p}=75$ & Higher inlet compression (MAX-1: $\left.r_{p}=70\right)$ \\
$M_{\text {design }}=8$ & Designed for lower Mach number (MAX-1: $\left.M_{\text {design }}=9\right)$ \\
$M_{\text {robust }}=2$ & Inlet less robust to changes in Mach number (MAX-1: $\left.M_{\text {robust }}=4\right)$ \\
$\alpha_{\text {robust }}=0$ & Inlet less robust to changes in angle of attack $\left(\mathrm{MAX}-1: \alpha_{\text {robust }}=1.5^{\circ}\right)$ \\
$\psi_{E}=5.73^{\circ}$ & Increased dihedral angle (MAX-1: $\left.\psi_{E}=2.86^{\circ}\right)$ \\
$n_{\text {ext }}=1$ & Single-ramp inlet (MAX-1: $\left.n_{\text {ext }}=2\right)$ \\
\hline \hline
\end{tabular}

Table 3. Trajectory fuel consumption and efficiency for several vehicle designs.

\begin{tabular}{lcccc}
\hline \hline Vehicle & Initial mass & Initial fuel mass & Fuel used & Trajectory $\boldsymbol{I}_{s p}$ \\
\hline MAX-1 & $30355 \mathrm{~kg}$ & $11610 \mathrm{~kg}$ & $5881 \mathrm{~kg}$ & $1021 \mathrm{~s}$ \\
$r_{f}=0.6$ & $32677 \mathrm{~kg}$ & $13932 \mathrm{~kg}$ & $6349 \mathrm{~kg}$ & $1017 \mathrm{~s}$ \\
$\Delta x_{c g}=0$ & $30355 \mathrm{~kg}$ & $11610 \mathrm{~kg}$ & $5617 \mathrm{~kg}$ & $1074 \mathrm{~s}$ \\
$\Delta x_{c g}=0.025$ & $30355 \mathrm{~kg}$ & $11610 \mathrm{~kg}$ & $5617 \mathrm{~kg}$ & $1041 \mathrm{~s}$ \\
$r_{p}=75$ & $30246 \mathrm{~kg}$ & $11569 \mathrm{~kg}$ & $5462 \mathrm{~kg}$ & $1103 \mathrm{~s}$ \\
$M_{\text {design }}=8$ & $30434 \mathrm{~kg}$ & $11400 \mathrm{~kg}$ & $5500 \mathrm{~kg}$ & $1102 \mathrm{~s}$ \\
$M_{\text {robust }}=2$ & $30213 \mathrm{~kg}$ & $11556 \mathrm{~kg}$ & $5347 \mathrm{~kg}$ & $1128 \mathrm{~s}$ \\
$\alpha_{\text {robust }}=0^{\circ}$ & $30844 \mathrm{~kg}$ & $11797 \mathrm{~kg}$ & $5517 \mathrm{~kg}$ & $1115 \mathrm{~s}$ \\
$\psi_{E}=5.73^{\circ}$ & $30355 \mathrm{~kg}$ & $11610 \mathrm{~kg}$ & $5872 \mathrm{~kg}$ & $1022 \mathrm{~s}$ \\
$n_{\text {ext }}=1$ & $37204 \mathrm{~kg}$ & $14230 \mathrm{~kg}$ & $8081 \mathrm{~kg}$ & $897 \mathrm{~s}$ \\
\hline \hline
\end{tabular}

with a higher mass $\left(r_{f}=0.6\right)$ required substantially more fuel mass to fly the trajectory but showed only a small decrease in the trajectory specific impulse.

\section{Design Optimization}

Using Table 3 it is possible to select a smaller set of design variables for which to perform an optimization. Two of the parameters that had a large effect on the specific impulse were design Mach number $\left(M_{\text {design }}\right)$ and robustness to changes in Mach number $\left(M_{\text {robust }}\right)$.

Figure 8 shows a contour of the trajectory specific impulse for a range of possible designs constructed by changing these two parameters. Many of the possible geometries were not able to fly the entire trajectory, and the resulting constraints are shown by black curves in Fig. 8.

In this work, a grid of possible designs (shown by purple and green points in Fig. 8) were considered individually, and the optimization was performed on a surrogate constructed from those results. It was found that the optimum vehicle design was one with no designed robustness to Mach number and designed for a Mach number in the middle of the trajectory (large green circle in Fig. 8).

A comparison of the baseline and optimized vehicle geometries is shown in Fig. 9, and an overlay of the two inlet designs is shown in Fig. 10. From Fig. 10 it can be seen that the optimized inlet has steeper inlet ramp angles. As a result, that design has more lift from the inlet, and the vehicle can fly at a lower angle of attack. Therefore even though the inlet may have less efficient operation, the total vehicle performance is higher due to the reduced drag resulting from the lower angle of attack.

To separate the effects of inlet robustness and inlet compression, Fig. 11 contains designs with varying design Mach number, robustness, and inlet compression ratio. Designs with the same maximum design 


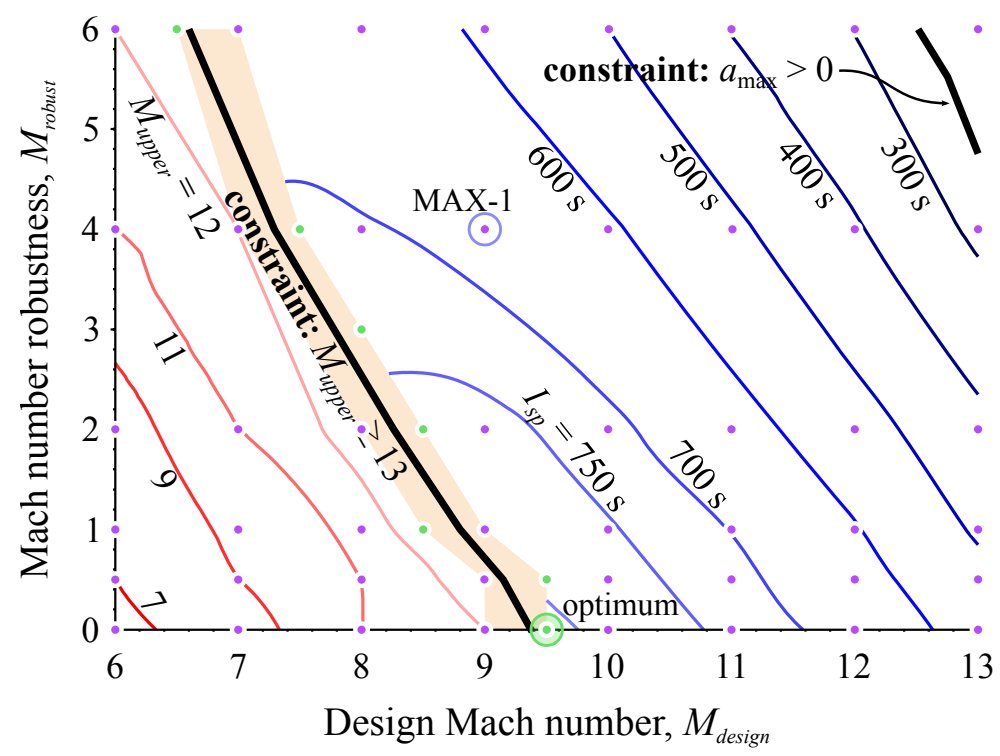

Figure 8. Contours of rocket-equivalent specific impulse $\left(I_{s p}\right)$ in blue. Points used for surrogate are shown in green and purple. The optimum feasible design $\left(M_{\text {design }}=9.5, M_{\text {robust }}=0\right)$ is circled.

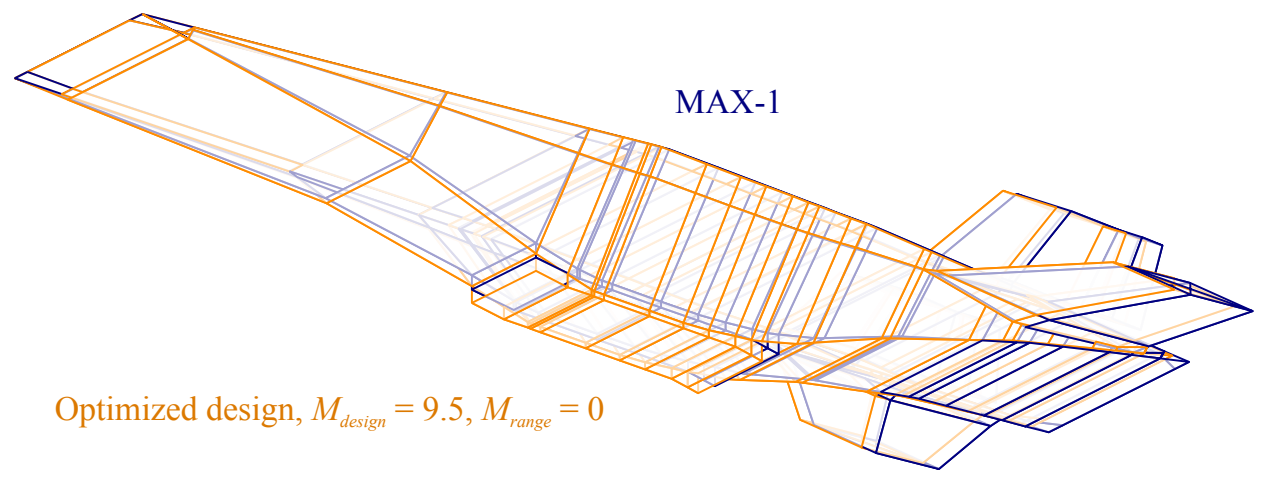

Figure 9. Optimized vehicle result overlayed with MAX-1.

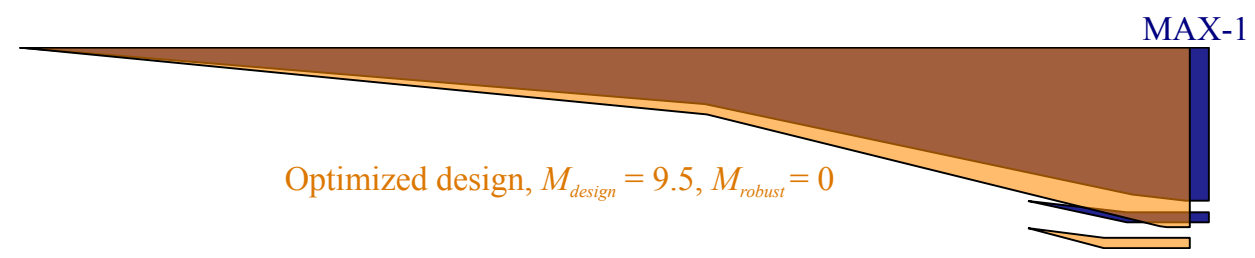

Figure 10. Comparison of MAX-1 and optimized inlet designs.

Mach number $\left(M_{\max }\right)$ are given the same symbol, and designs with the same inlet compression ratio (when flying at a Mach number equal to $M_{\max }$ ) have matching colors. The remaining design parameter, which is the minimum design Mach number $\left(M_{m i n}\right)$, is the horizontal axes of Figs. 11a and 11b.

Figure 11a shows that even when the effect of robustness on compression is properly accounted for (designs with the same $r_{p}$ and $M_{\max }$ values have the same compression at the same Mach number), increases robustness is associated with slightly decreased performance. This is somewhat difficult to see from the figure, but designs further to the right are designed for less robustness. For example, triangles with $M_{\min }=12$ 


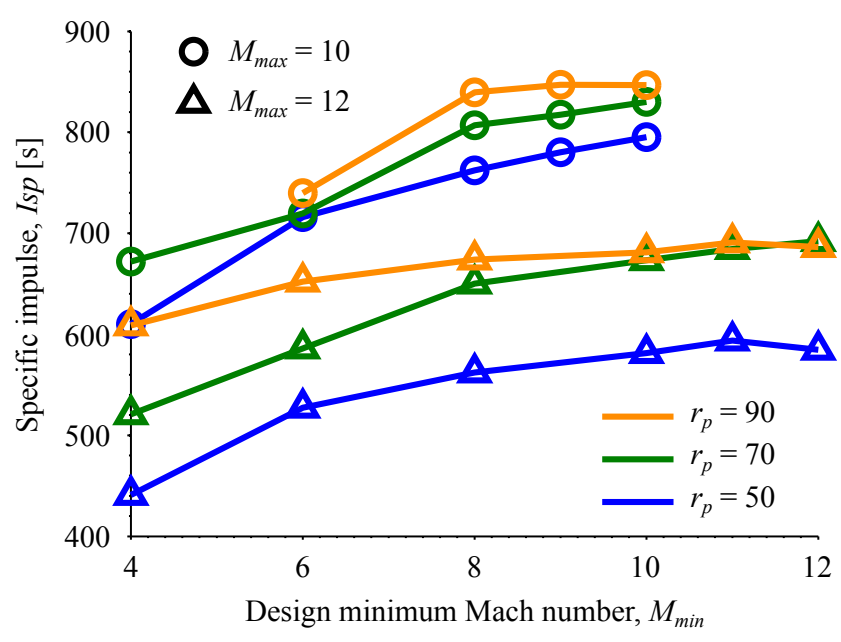

a) First set of designs with varying compression ratio $\left(r_{p}\right)$.

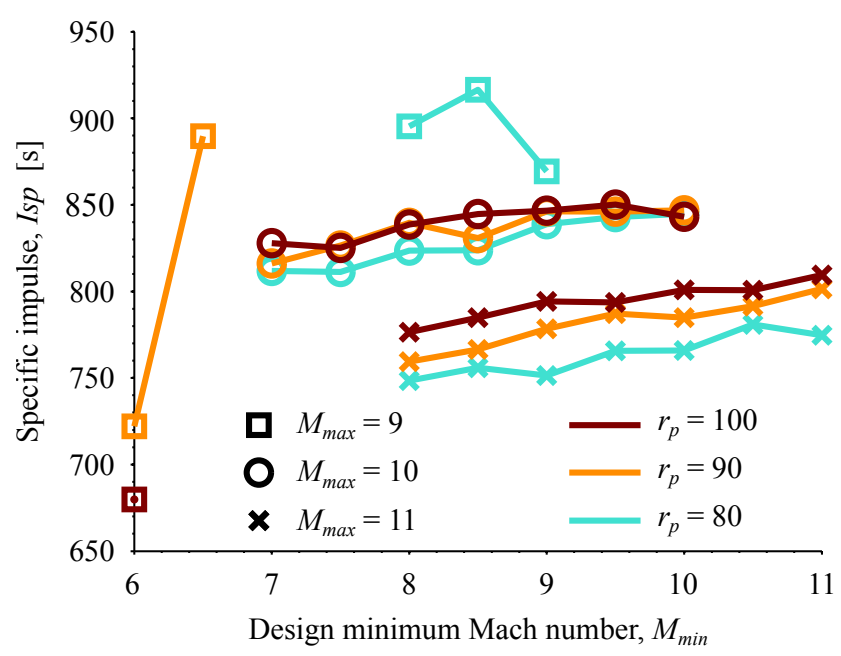

b) Refined design set.

Figure 11. Rocket-equivalent specific impulse $\left(I_{s p}\right)$ for designs with varying design Mach numbers and compression ratio.

and circles with $M_{\min }=10$ have no design robustness. However, unlike in Fig. 8, the effect of robustness is much weaker than the effect of the other two design variables. For some of the curves, a slightly robust inlet performs better than the $M_{\min }=M_{\max }$ design. As far as selecting new designs to further increase the performance, the trends from Fig. 11a are that

1. lower values of $M_{\max }$ are favorable;

2. a small (or zero) amount of robustness yields the highest specific impulse;

3. higher compression is usually favorable; and

4. high compression decreases the chances that the design can fly the whole trajectory.

This led to the selection of second set of values for the three design variables. The values for the maximum design Mach number $\left(M_{\max }\right)$ are 9, 10, and 11. The lowest of these, $M_{\max }=9$, is the most interesting because Fig. 8 shows that such designs are very close the constraint where the vehicle either can or cannot fly the trajectory to Mach 13. The compression ratio varies from 80 to 100, using 100 as an upper constraint as it roughly corresponds to a combustor entrance static pressure of $2 \mathrm{~atm}$. 
Fig. $11 \mathrm{~b}$ shows that only a few of the $M_{\max }=9$ designs that were tried successfully flew the trajectory, but these included the four highest-performing designs. Although the general trend was that efficiency increased with higher compression ratios $\left(r_{p}\right)$, the constraints led to a design with lower compression being the best performer. Compared to the performance of the original MAX-1 design, the specific impulse has increased from $660 \mathrm{~s}$ to $949 \mathrm{~s}$. Of the three design variables considered, the optimized design had none equal to the minimum or maximum value allowed. However, there was an active constraint; a slight increase in inlet compression or a slight decrease in $M_{\max }$ would lead to a vehicle that could not fly the trajectory. It appears from the cases investigated here that this type of constraint is very likely to be active when optimizing a scramjet-propelled vehicle. Because the connected curves in Fig. 11 are relatively flat, it appears that an inlet that avoids detrimental shock interactions over much but not all of the trajectory is favorable when practical considerations are made. Such considerations include atmospheric variation, ability to fly slightly altered trajectories, and, most importantly, model uncertainty. Some of the conclusions regarding the specific design are strongly dependent on the trajectory and mission being considered, which is statement typical of most aerospace vehicles.

\section{Dual-Mode Considerations}

In order to operate at lower Mach numbers, conceptual air-breathing hypersonic vehicles often have dualmode engines. In addition to the scram mode (supersonic combustion ram mode) considered in the previous sections, these engines can operate in the ram mode, in which the flow speed is less than the local speed of sound where the fuel is injected. Although the distinction between subsonic combustion and supersonic combustion may seem like a subtle one, it can have a profound effect on performance. For the class of engine geometries considered here (see Fig. 2b), the MASIV model predicts a discontinuous performance at the point of transition between these two modes.

While designing an engine geometry that avoids or attenuates this behavior is perhaps essential for an operational vehicle, there is also the question of how to design a trajectory for a vehicle with it. In the case of the MAX-1 vehicle (and those with related engine geometries consisting of a single fuel injection port and a constant-area combustion section followed by an expanding section), the thrust is about $25 \%$ higher in ram mode than scram mode (for the same amount of fuel).

Figure 12 shows the result of two trajectory optimizations (for a fixed vehicle) with the constraint of constant dynamic pressure removed. Both trajectories accelerate from Mach 4.5 to 7.0, and both contain a transition from ram mode to scram mode (labeled $M_{R S}$ in Fig. 12). The difference between the two trajectories is that one of them, trajectory $A$, has unconstrained altitude at both the start $(M=4.5)$ and end $(M=7.0)$ points while trajectory $B$ is constrained to have a dynamic pressure of $100 \mathrm{kPa}$ at the start and end points.

While the engine ostensibly performs better at lower altitudes in both modes, both optimized trajectories contain segments of flight at higher altitudes (equivalently, lower dynamic pressures). The reason for this behavior is to prolong the period of ram-mode operations. Considering two flight conditions with the same Mach number but different altitudes, the higher-altitude condition results in less air entering the engine, and thus thermally choking is more likely to occur. By prolonging the ram-mode operation, the overall fuel consumption decreases, even though at the lower Mach numbers ram-mode flight at lower altitude would be more efficient. Among other things, this means that the optimized trajectory to accelerate from Mach 4.5 to Mach 5 would not be a segment of either trajectory $A$ or $B$. Another interesting feature is that ram-scram transition occurs during a dive in both optimized trajectories.

The primary reason for including these results is to illustrate how sensitive the result of a design optimization can be to the choice of objective function for air-breathing hypersonic vehicles. Scram-only trajectories are typically much easier to analyze, but most practical scramjet vehicles are likely to have dualmode engines. Therefore, although the general statements about inlet design may be useful for hypersonic vehicles in general, the trajectory optimization problem considered in this section is more representative of the considerations that will apply to an operational vehicle. 


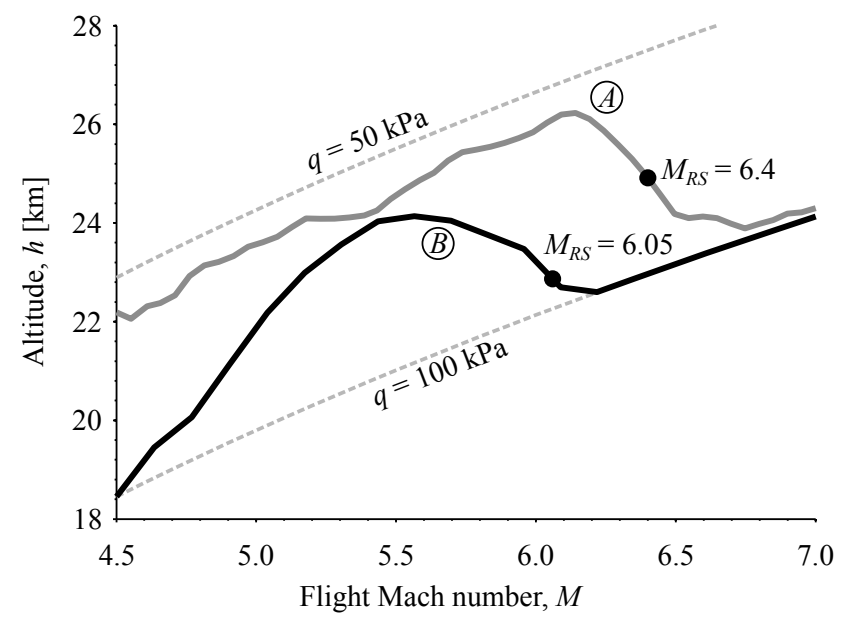

a) Altitude $(h)$. Ram-scram transition is marked for both trajectories.

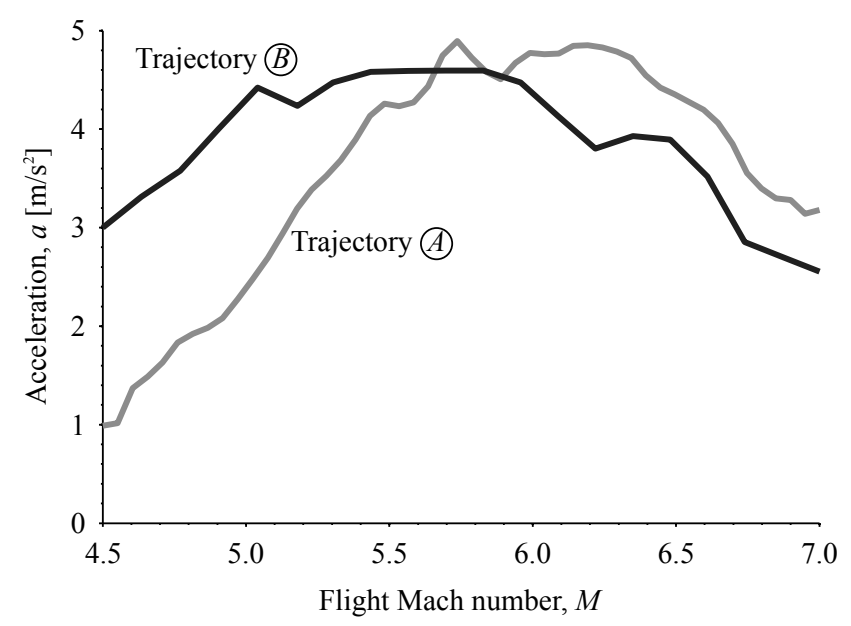

b) Acceleration (a).

Figure 12. Comparison of two optimized trajectories without constant dynamic pressure constraint. The initial $(M=4.5)$ and final $(M=7)$ dynamic pressures are constrained to $100 \mathrm{kPa}$ for trajectory $B$.

\section{Conclusions}

Design optimization is performed for an air-breathing hypersonic vehicle simultaneously considering geometric design and acceleration as decision variables. Optimum trajectories were shown to favor high accelerations, which can be limited for two different reasons. At lower Mach numbers, the maximum acceleration is largest acceleration that can be maintained without causing thermal choking in the combustor. At high Mach numbers, the optimization selects the acceleration such that all of the oxygen available that enters the engine is consumed. This often requires injecting fuel at equivalence ratios much higher than unity, and combustion efficiencies decrease as the Mach number increases.

Designs that have a high contribution of lift in the inlet were shown to improve overall fuel efficiency of the vehicle for an ascent trajectory from Mach 7 to Mach 13. The optimized inlet geometry was one with inefficient performance at the lowest and highest Mach numbers but very high performance around Mach 8 to 10. However, some of the non-robust designs were not able to fly the prescribed trajectory. This suggests an intricate trade off between robustness, performance, and feasibility.

In addition, an introductory look at trajectory optimization for dual-mode engines is presented. An 
interesting aspect of the resulting trajectory is that its ram-mode and scram-mode phases would not be optimal for either a ram-only or scram-only trajectory, respectively. Instead, the optimizer made a strategic decision to select a trajectory that kept the vehicle in ram-mode longer to enhance total fuel consumption at the expense of instantaneous fuel consumption. Such counterintuitive results are likely to be typical of optimization applied to air-breathing hypersonic vehicles.

\section{Acknowledgements}

This research is funded by the Air Force Research Laboratory/Air Vehicles Directorate grant FA 8650-072-3744 for the Michigan/AFRL Collaborative Center in Control Sciences (Michael Bolender as technical monitor).

\section{References}

[1] Calise, A. J., Corban, J. E., and Flandro, G. A., "Trajectory optimization and guidance law development for national aerospace plane applications," American Control Conference, 1988, pp. 1406-1411.

[2] Corban, J. E., Calise, A. J., and Flandro, G. A., "Rapid Near-Optimal Aerospace Plane Trajectory Generation and Guidance," Journal of Guidance, Vol. 14, 1991, pp. 1181-1190.

[3] Powell, R. W., Shaughnessy, J. D., Cruz, C. I., and Naftel, J. C., "Ascent Performance of an Air-Breathing Horizontal-Takeoff Launch Vehicle," Journal of Guidance, Vol. 14, No. 4, 1991, pp. 834-839.

[4] Olds, J. R. and Budianto, I. A., "Constant Dynamic Pressure Trajectory Simulation with POST," Aerospace Sciences Meeting and Exhibit, 1998, AIAA Paper 1998-0302.

[5] McGinnis, P. M., "Robust Hypersonic Conceptual Design Trajectory Techniques for PMDO Applications Using POST," 41st AIAA/ASME/SAE/ASEE Joint Propulsion Conference \& Exhibit, 2005, AIAA Paper 2005-4423.

[6] Lu, P., "Inverse Dynamics Approach to Trajectory Optimization for an Aerospace Plane," Journal of Guidance, Control, and Dynamics, Vol. 16, 1993, pp. 726-732.

[7] Lu, P., "Analytical Solutions to Constrained Hypersonic Flight Trajectories," Journal of Guidance, Control, and Dynamics, Vol. 16, 1993, pp. 956-960.

[8] Bowcutt, K. G., "Multidisciplinary Optimization of Airbreathing Hypersonic Vehicles," Journal of Propulsion and Power, Vol. 17, 2001, pp. 1184-1190.

[9] Lewis, M. J., "Significance of Fuel Selection for Hypersonic Vehicle Range," Journal of Propulsion and Power, Vol. 17, No. 6, 2001, pp. 1214-1221.

[10] Bing, C., Xu, X., and Guobiao, C., "Single- and Multi-objective Optimization of Scramjet Components Using Genetic Algorithms Based on a Parabolized Navier-Stokes Solver," 42nd AIAA/ASME/SAE/ASEE Joint Propulsion Conference \& Exhibit, 2006, AIAA Paper 2006-4686.

[11] Theisinger, J. E. and Braun, R. D., "Multiobjective Hypersonic Entry Aeroshell Shape Optimization," 12th AIAA/ISSMO Multidisciplinary Analysis and Optimizaition Conference, 2008, AIAA Paper 2008-5873.

[12] Grant, M. J., Clark, I. G., and Braun, R. D., "Rapid Simultaneous Hypersonic Aerodynamic and Trajectory Optimization Using Variational Methods," AIAA Atmospheric Flight Mechanics Conference, 2011, AIAA Paper 2011-6640.

[13] Smart, M. K., "Optimization of Two-Dimensional Scramjet Inlets," Journal of Aircraft, Vol. 36, No. 2, 1999, pp. 430-433.

[14] Dalle, D. J., Fotia, M. L., and Driscoll, J. F., "Reduced-Order Modeling of Two-Dimensional Supersonic Flows with Applications to Scramjet Inlets," Journal of Propulsion and Power, Vol. 26, No. 3, 2010, pp. 545-555.

[15] Torrez, S. M., Driscoll, J. F., Dalle, D. J., and Fotia, M. L., "Preliminary Design Methodology for Hypersonic Engine Flowpaths," 16th AIAA/DLR/DGLR International Space Planes and Hypersonic Systems and Technologies Conference, 2009, AIAA Paper 2009-7289.

[16] Brown, M., Mudford, N. R., Neely, A. J., and Ray, T., "Robust Optimization of Two-Dimensional Scramjet Inlets," 14th AIAA/AHI Space Planes and Hypersonic Systems and Technologies Conference, AIAA Paper 20068140, 2006. 
[17] Dalle, D. J., Torrez, S. M., and Driscoll, J. F., "Performance Analysis of Variable-Geometry Scramjet Inlets Using a Fundamental Model," 47th AIAA/ASME/SAE/ASEE Joint Propulsion Conference and Exhibit, 2011.

[18] Torrez, S. M., Driscoll, J. F., Ihme, M., and Fotia, M. L., "Reduced Order Modeling of Turbulent Reacting Flows With Application to Scramjets," Journal of Propulsion and Power, Vol. 27, No. 2, March-April 2011, pp. 371-382.

[19] Dalle, D. J., Torrez, S. M., and Driscoll, J. F., "Rapid Analysis of Scramjet and Linear Plug Nozzles," Journal of Propulsion and Power, Vol. 28, No. 3, 2012, pp. 545-555.

[20] Dalle, D. J., Torrez, S. M., and Driscoll, J. F., "Turn Performance of an Air-Breathing Hypersonic Vehicle," AIAA Atmospheric Flight Mechanics Conference, 2011.

[21] Dalle, D. J., Torrez, S. M., Driscoll, J. F., and Bolender, M. A., "Flight Envelope Calculation of a Hypersonic Vehicle Usinga First Principles-Derived Model," 17th AIAA International Space Planes and Hypersonic Systems and Technologies Conference, 2011, AIAA Paper 2011-2394.

[22] Vick, T. J., Muse, J. A., and Bolender, M. A., "A Hypersonic Vehicle Model Generator for MASIV," AIAA Modeling and Simulation Technologies Conference, 2012.

[23] “U.S. Standard Atmosphere,” U.S. Government Printing Office, 1976, Washington, D.C. 